https://doi.org/10.48009/1_iis_2010_237-246

\title{
WEB COMMUNICATIONS STRUCTURE
}

\author{
James J. Lee, Seattle University, leej@seattleu.edu \\ Ben B. Kim, Seattle University, bkim@ seattleu.edu \\ Ted E. Lee, University of Memphis, elee@memphis.edu \\ Pairin Katerattanakul, Western Michigan University, pairin@wmich.edu \\ Wan Yui See, New York University, wys207@nyu.com
}

\begin{abstract}
The purpose of this study is to propose a classification scheme of Web communication. Because it is the most widely used platform of the Internet communication, the Web creates emerging global databases or information systems. Web communication structure is investigated as differentiated elements that can be recognized in the patterned communication flows on the Web. Eight structures are identified and proposed structural analysis can contribute to examine how businesses on the Web attempt to control the interactions with users for the purpose of communicating their intentions.
\end{abstract}

Keywords: the web, communication structure, communicators, mode of communications, control scheme, patterned communications

\section{INTRODUCTION}

The communication structure of the Internet is different from its physical world counterparts. In the physical world, communication structure is defined as the 'differentiated elements that can be recognized in the patterned communication flows in a system' (Rogers and Kincaid, 1981, p. 146). Rigorous researches have formulized linear models of communication (Shannon and Weaver, 1949) and small groups of communication (Leavitt, 1951; Shaw, 1954) have evolved to complex communication structure and social network analysis (Rogers and Kincaid, 1981; Scott, 1991; Wasserman and Faust 1994). However the communication dimensions identified by these theories remain consistent as they are all based on human interactions. Thus, a basic assumption of communication structure from social network analysis has been the face-to-face or related communications component between humans.

Network Technology (IT \& networking technology), especially on the Internet, however, provides new communication dimensions in time and space (reach real time information instantly), which have been uncontrollable in the physical world. The Internet communications create new opportunities such as synchronous (videoconferencing) or asynchronous (groupware) communications. In addition to these opportunities, IT/IS delivers processed contents, which makes digital contents rich. Lately, companies have realized the importance of content management as they implement e-business practice everywhere, such as B2B, B2C, B2E, C2C, G2C, G2B, and G2G. As the Web becomes a new standard platform of modern communication, identifying the web communication structure illuminates how to control time and space dimensions in the final consumption of communication by humans.

World Wide Web has enhanced the experiences of the Internet communications even better. As becoming a new platform, the Web provides a uniform but platform-independent environment. The success of the Web has extended its territory from static web to dynamic web such as Web 2.0. Therefore, entire information on the Web is shared, at the same time; the culture of the Web is self-organized (O'Mahony and Ferraro, 2007). This nature of the Web is originated from the core of the Internet, open source. The Internet technology has been developed by open source since network protocol software is freely distributed (Campbell-Kelly, 2008). Any web site are fundamentally public unless private organizations want to build intranet or extranet. Communication experiences through the Web, therefore, is inherited the open source culture. It is not surprising that you would see other readers' reviews in Amazon.com.

The purpose of this study is to propose a classification scheme of Web communication in business domains. It addresses the role of classification of digital documents in business organization contexts on the Web. It contributes to the understanding of change and the socioorganizational enactment of processes of classification of digital documents by classifying Web communications. Because it is the most widely used platform of the Internet communication, the Web creates emerging global databases or information systems. As IT shifts its focus to connected and integrated environments, we face the 
new era of communication with processed contents on the Web. We, therefore, define the communication practice on the Web between humans and web sites as 'web communication structure' in this study where web communication structure is defined as 'differentiated elements that can be recognized in the patterned communication flows on the Web' (modified from Rogers and Kincaid, 1981, p. 146).

\section{PATTERNED COMMUNICATION FLOWS ON THE WEB}

Originally developed in the context of management of file structure, World Wide Web (WWW) technology is based on Hypertext Markup Language (HTML). The concept of hypertext is not quite original, since there had been theories from physicists such as Albert Einstein who used terms such as hyperspace, worm hole, black hole, and time machine. The WWW, however, is purely evolved from hypertext in the late 1990s. Using network for transmission and address scheme and servers for storage, the texts (hypertext or hyperlink as appeared on web pages) jumping around computers across the world have brought us to invaluable experiences, despite the texts having been prepared beforehand. What we have experienced is a small scale time machine in printing mode - markup language having originated from the printing industry. With the concept of "book" articulated as knowledge accumulation, the WWW has been rapidly adopted by society as a virtual knowledge repository (virtual book).

The text-based webs were limited in that contents can be published as static webs. The early static webs have evolved rapidly into dynamic webs, and then active server pages (ASPs). These evolutions resulted from the preference of users to the logic of the WWW hypertext. But we desired to extend the role of WWW beyond simple information exchange. Since web browsers are running from computing systems, it is easy to develop "programming" capability on web pages. Thus, current web sites boast their feel and look of stand-alone application with convenient user interfaces. This result in WWW development as global databases and information systems of front-end integration aligned with backend integration such as Enterprise Resource Planning (ERP), Supply Chain Management (SCM), and Customer Relationship Management (CRM).

With the movement to the next level of web, Web 2.0 (O'Reilly, 2005) and the Semantic Web (Berners-Lee et al., 2001; Shadbolt et al., 2006) are being developed and used. Though there are differences in technical and theoretical approaches, both Web 2.0 and the Semantic Web extend the Web's capability to supporting the inference model as well as the reference model. Using hyperlinks on the web pages naturally shapes web communication under the reference model. As you navigate the Web to search certain information, you need to refer to more and more web sites. Because current web applications can be backed by databases and information systems, the Web can provide more refined information using the inference engine. Thus, a trusted web can display not only processed and updated information of its own internal source, but also aggregated trustworthy information of related external sources without navigating each web site. XML based Web Service (Sayah and Zhang 2004) shows the concept of reference model while Really Simple Syndication (RSS) indicates the inference model.

The Web serves fundamentally as a navigating system that allows human users to actively browse different sites on the Internet. With recent advancement in communication scheme, the Web provides rich information and communication tools. Tools being the most commonly used as communication moderators by web domains were identified. In this study, we categorized them in terms of the browsing experience into three communication factors; 1) types of communicators, 2) modes of communications, and 3) control scheme

\section{Types of communicators}

The first Internet browsing experiences were provided to users through the use of hypertext-based websites. Recently, web systems have advanced user's browsing experience by providing a humancomputer interaction through the usage of application and software tools. As each web site is composed of files that reside in the domain's web server, domain name systems (DNS) in the form of Uniform Resource Locators (URLs) are used to identify web resources and to locate the physical web site. As a result, users could recognize each web site as a 'name' (for example, amazon.com), with which they can objectively communicate with. Hence, the two types of communicators in web communication structure are; 1) users (humans) who interact with the web sites directly and 2) web servers who are referred or inferred by users and other server.

\section{Modes of communications}

Modes of communications on the Web pertain to the 
creation of communication structure between source(s) and target(s) communicators. Due to the advancement of IT, web environments can connect multiple source and target communicators together easily. Advanced web technologies such as XML, web services, and web programming, have evolved the web platform into a medium of complex communication structure. Thus, features like users' comments on Amazon.com or price comparisons on BizRate.com can now be effectively processed and efficiently shared. These examples highlight the different modes of complex communication structure that are entailed in websites to allow efficient and multifaceted client/server interaction. This client/server architecture evolved from the initial one-to-one relationship format to multidirectional relationships between numerous sources and targets with the introduction of IT/IS. As a result, web site can be engaged by a single user or multiple users asynchronously while web servers can be related by reference or inference engines.

\section{CONTROL SCHEME}

While the structure of each 'mode of communication' is determined by the different relationship between communicators, the structure of a 'control scheme' is determined by the moderating effects used to determine efficient communication. Control scheme simply leverages the direction of who signifies, legitimates, and dominates the modes of communications (Giddens, 1984). For example, while users can search and inquire for precise information hence dominating the communication direction, they can also take a more passive role and allow the Web to guide them to a series of less specific information leads.

\section{RESEARCH PROCEDURE}

The World Wide Web has a tremendous number of sites, and each of these web sites may all take on a different role. This reflects that a web site is flexible enough to create fresh opportunities in communication structure as a web site is produced, reproduced, and transformed (Orlikowski, 1991). In this study, we limited the scope of analyses to just web sites in the business domain, specifically those that provide services to its visitors. This was because web site that provides services often has clear goals and missions. They also host up-to-date web technologies to increase its attractiveness to lure more visitors.

A total of 71 different business domain web sites were selected and examined in 2007. Various websites were analyzed according to their genre. Similar websites with the same usage/function were grouped together to form a "category". There were a total of 19 categories found from the 71 web sites investigated (Table 3). More categories could have been derived from the numerous forms of web sites currently in existence, but the 19 categories represents a significant cross-section and provides a sufficient number of instances to examine the web communication structure (Glaser and Strauss, 1967; Glaser 1978). The three communication structure factors explained above were not considered at the initial investigation but were deduced through categorization of the web sites (Strauss and Corbin, 1998; Turner, 1981).

One web site was selected from each category to act as the category's sample representative. The 19 categories are listed in Table 3, "Web communication structure", under the column "Category Number". We analyzed each web site based on two factors: contents and results. Contents were examined by identifying the types of communicators and modes of communications. Results were investigated in response to the interaction between the user and the web site to reveal which control scheme make up the web communication structure. Therefore, web communication structure is determined when a user is using the Web. Contents (C) determines the relationship between sources (user) and targets (web server) communicators, while Results (R) determines the control scheme that explains which communicator dominates the web interaction. The contents and results gathered from all of the 19 categories were then compared against each other for similarities and differences in the aforementioned three factors. Coding numbers were identified and developed to help differentiate the diverse results for $\mathrm{C}$ and $\mathrm{R}$ into sub categories (3.1. and 3.2) and then organized into groups according to similarity in communication patterns. This results in a distinguishing pattern type for each category as discussed in the Section 4.

It must be noted that due to the characteristic and the prominent usage for category 4 (organizational web sites), the category was further divided into two separate sub-categories, $4 \mathrm{a}$ and $4 \mathrm{~b}$. The individual category evidence provided later in the paper gives a specific overview for the similarities and differences between the two groups. It must be noted that although both $4 \mathrm{a}$ and $4 \mathrm{~b}$ belong to the same umbrella group, their target goals and forms of communication method to achieve these goals set them apart. They were thus identified as having different patterns of communication and were coded in terms of their 
method of information presentation to their target audience which were either logic (fact) driven or subject (customer) driven.

The rest of section 3 explains the coding scheme. Each code for $\mathrm{C}$ represents a combination of 2 cases of communicators types (one, many) and 2 cases of modes of communications (reference, inference). Each code for $\mathrm{R}$ represents a different control scheme classification. Categories which had similar C-R coding patterns were put together. The definition of each classification is given below.

\section{Coding scheme: Contents}

This study did not put consideration into human to human synchronous communication (real time chatting or email communication) on the Web. The focus was on asynchronous web communication between human users and web sites. For the purposes of the study, a human user who uses a web browser is considered as the source of communication while a web site that the human user is visiting is the target of communication.

Because of the ability of web sites to share information among users in an asynchronous way (customer reviews of products), the source of communication can be from a single or multiple user(s). Similarly, web servers can host contents from either a single or multiple web server(s). Thus, how the target web server relates to other web servers can be identified as either providing a 'reference model', focusing on providing contents as evidences; or as an 'inference model', emphasizing on imposing meanings of contents. This is summarized as below:

Table 1. Contents $(\mathrm{C})$ coding scheme

\begin{tabular}{|c|l|l|}
\hline Code & \multicolumn{1}{|c|}{ Name } & \multicolumn{1}{c|}{ Definition } \\
\hline $\mathbf{1}$ & UniLateral & $\begin{array}{l}\text { Single source } \\
\text { reference model }\end{array}$ \\
\hline $\mathbf{2}$ & UniPlex & $\begin{array}{l}\text { Single source } \\
\text { inference model }\end{array}$ \\
\hline $\mathbf{3}$ & MultiLateral & $\begin{array}{l}\text { Multi source } \\
\text { reference model }\end{array}$ \\
\hline $\mathbf{4}$ & MultiPlex & $\begin{array}{l}\text { Multi source } \\
\text { inference model }\end{array}$ \\
\hline
\end{tabular}

\section{Coding scheme: Results}

Interaction plays a large role throughout communication and so was included as part of the control scheme where it was categorized according to the degree a user can tailor the contents a web site presents. Due to web sites applying many IS, such as querying and processing data, the interactivity of web communication through the results displayed based on users' concerns play major roles in today's web environment. Based on the nature of how user's concerns are addressed, the results (contents upon users' preferences) are summarized as below:

Table 2. Results $(\mathbf{R})$ coding scheme

\begin{tabular}{|c|l|l|}
\hline Code & Name & \multicolumn{1}{|c|}{ Definition } \\
\hline $\mathbf{1}$ & $\begin{array}{l}\text { Logic- } \\
\text { driven }\end{array}$ & $\begin{array}{l}\text { information does not pertains to } \\
\text { or reflect the user's specific } \\
\text { preferences, but it follows the } \\
\text { logic provided by web sites }\end{array}$ \\
\hline $\mathbf{2}$ & $\begin{array}{l}\text { Subject- } \\
\text { driven }\end{array}$ & $\begin{array}{l}\text { information pertains to or } \\
\text { reflects the user's specific } \\
\text { preferences, thus it follows the } \\
\text { subjects of user's interest }\end{array}$ \\
\hline
\end{tabular}

\section{WEB COMMUNICATION STRUCTURE}

The patterns of communication flows on the Web identified for each category are summarized in Table 3. The eight main classification structures were derived by identifying the four contents codes associated with the two results codes. This classification is presented in the proceeding sections (4.1 through 4.4) through a revision for the communicator's relationship followed by a presentation of control schemes in logic and subject driven cases.

Table 3. Web communication patterns

\begin{tabular}{|c|c|c|}
\hline \multicolumn{2}{|c|}{ Pattern } & \multirow[b]{2}{*}{ Category Number } \\
\hline $\mathbf{C}$ & $\mathbf{R}$ & \\
\hline 1 & 1 & $\begin{array}{l}\text { (3) Tracker Sites } \\
\text { (15) Online Planner/Interactive Schedule }\end{array}$ \\
\hline 1 & 2 & $\begin{array}{l}\text { (1) Information/Dictionary/Encyclopedia } \\
\text { (8) Newspaper/Magazine } \\
\text { (13) Help and Support } \\
\text { (16) Interactive Learning and Response } \\
\text { (17) Reference Sites }\end{array}$ \\
\hline 2 & 1 & (2) Personal/Self Expression Sites \\
\hline 2 & 2 & $\begin{array}{l}\text { (7) Database/Archives/Collection } \\
\text { (18) Shopping/Auctions }\end{array}$ \\
\hline 3 & 1 & (4a) Organizational Websites \\
\hline 3 & 2 & (4b) Organizational Websites \\
\hline 4 & $\mathbf{1}$ & $\begin{array}{l}\text { (9) Deals/Savings Websites } \\
\text { (11) Downloads/Torrent } \\
\text { (12) Forums }\end{array}$ \\
\hline
\end{tabular}




\begin{tabular}{|c|c|l|}
\hline & & $\begin{array}{l}\text { (14) Review/Critiques } \\
\text { (19) Advertisement }\end{array}$ \\
\hline $\mathbf{4}$ & $\mathbf{2}$ & $\begin{array}{l}\text { (5) General Search Engine Sites } \\
\text { (6) Specific Search Sites } \\
\text { (10) Entertainment Websites }\end{array}$ \\
\hline
\end{tabular}

\section{Unilateral (one-to-one)}

The communication relationship is unilateral as it involves only one source and one target. The information displayed to the source is referred solely by the target. Although additional target sources may be indicated using 'Links', communication is fundamentally between the one-to-one interaction of the source and the target.

\section{Unilateral Logic-driven (C1R1): http://www.gottem.net}

The function of this target is to track and indicate a visit $\log$ for a particular website of the source. The target displays the visit log allowing the source to use the information as reference but does not provide any other information. In most cases, the site monitored would usually be regulated by the source. However if the source wants to gain additional information, they will have to acquire it through other means.

Contents: Information is displayed as soon as login is completed. The name of the target is shown (Got'em). Option situated along the top of the page allow navigation to "help", "contact", "login" panels. There are advertisements situated underneath. The middle portion of the page offers more navigation panels along with the visit $\log$. The bottom portion of the page show the tracking records which displays information including: the visitors user name, IP address, date and time of visit, the URL of viewed sites and the URL from which the visits were made from. In order for the source to acquire further information, navigation outside of the target would have to be made.

Results: The results are shown immediately after login presenting information gathered by Got'em previous to login. Manipulation of the information is not possible as they are at face-value. A few interactive examples include: the "sniffer" option which shows possible tracking devices when selected; an "insert" bar which allows web addresses to be imputed to check if they contain tracking devices; and the "create an account" options which when selected displays the form Got'em requires for member registration.

\section{Unilateral Subject-driven (C1R2):}

\section{http://support.microsoft.com}

The function of this target is to provide help and support for users of Microsoft products (primarily Window XP). The target shows a database which contains a collection of common errors. It allows searches for specific problems in addition to support requests. Help is also provided to diagnose problems in a step-by-step fashion with direction to additional assistance if needed.

Contents: The Microsoft logo is situated at the upper top portion of the page along with the location that is being browsed. Across from the logo is a searching bar with options on top that provides quick links to access different part of the page, or pages at a different location. On the left hand portion of the page are listing of different programs that can be chosen depending on particular inquiries. Included are programs such as Microsoft office, Microsoft outlook, windows XP, Internet Explorer, etc. Below the listing of programs are listings of different support options. Microsoft also provides international support. In the center portion of the page are listings of top issues and solution links, and underneath that are listing for new product supports. On the right hand portion of the page is a separate search tool that facilitates the finding of different support targets other than Microsoft.

Results: The results are very much tailored to the source. That is, different solutions are available for different types of problems. The results are usually displayed in procedural formation, assisting the source through step by step instructions. An interactive example is seen when "communications" was searched for using the search bar. Microsoft displayed a list of relevant results, and when a topic link was clicked on, Microsoft provided a solution to the particular topic problem indicated by the link.

\section{Uniplex (one-to-many)}

The communication relationship is uniplex as it involves only one source with multiple targets. This can happen when the source communicates with a target that infers information from other targets. The ultimate goal is to gather trustworthy information that matches the inquiry generated by the source. Communication is between the one-to-many interaction of the source to various targets attained through inference engines. 


\section{Uniplex Logic-driven (C2R1): http://www.xanga.com}

The target "Xanga,com" is an online "community" that is made up of personal blogs from member users. Xanga allows each member to have their own individualized site where they can personalize it in the way they prefer. Members can use their site as an online diary tool for expressing their thoughts and feelings; they have access to other member's site and have the ability to communicate with other members through the method of leaving "comments" and giving "props" on their blog entries. Unless specifically blocked, non-members can have access to any member sites, but only members within the xanga community can perform tasks such as creating new entries and leaving comments.

Contents: Members of the Xanga community goes to the main homepage of Xanga to log into their blog sites. On the top portion of the site, the "Xanga" logo is situated along with a search tool and different options that helps navigation around the target. Below these options are categories of different blog sites which the members can browse. They are separated into categories such as 'weblogs' (personalized sites), 'photos' (photos that members upload onto their sites), 'videos' (videos member uploaded), 'pulse', 'metros' , 'blogrings' (different members which have gather together by creating unique groups), and 'profiles' (the profile and personal information of the members). On the center left are the links to the categorized blogs by presented by the target and on the center right are advertisements and a tool showing a list of 'user's top rankings'.

Results: The results presented are very minimal even though there are a lot of options because it is the homepage. Login is required in order to access the real function of the target, which is accessing personal blogs. When viewing the pages of other members, sources do not have much influence on how the information on the page should be like; therefore, there is very little amount of interactions between the source and the target. On a member's blog page, the member's profile is situated on the left hand portion and an entry which spans from the middle to the right hand portion of the page. The layout of each page varies, depending on the preferences of the author. By scrolling down to the 'props' section of the entry, members can see comments other members has left to the author of the entry.

\section{Uniplex Subject-driven (C2R2):}

\section{http://www.ebay.com}

Ebay is an auction target which allows the posting of items for sale. However members must be register with the target to avoid the risk of scamming. The target gives its members the ability to both buy and sell items in an auction style format. Desired items can be searched through easily using pictures and descriptions provided by the seller. Trustworthiness is measured according to a "feedback" rating, which is provided by buyers or sellers from previous transaction experience through either positive or negative marks

Contents: Underneath the Ebay logo on the top left hand corner of the page is a search bar which allows an efficient locatation of items. In the center left hand portion of the page are different listings of links for specialty sites and also for different item categories. In the middle center of the page are Ebay advertisements for things either in popular demand or are on sale. On the right hand portion of the page are advertisements, and feature products displayed by an image and their genre. There are 'tap' options which indicates different Ebay categories (for example, motors), which searches product through different revenues.

Results: The result presented depends very much on the decisions and inquiries of the source as they browse the site. The product descriptions displayed would be on the products that the source is most interested in, either for selling or buying. There are many opportunities for different venues of navigation. When "laptop", an icon on the right hand portion of the page is clicked on, Ebay shows the different types of laptops along with a picture and a brief description of them. Most importantly, it shows the cost of the product if they were to make a purchase. To find more information about a particular item, the highlighted title would need to be selected after which a more product specific page would be available. When a search is made for the category regarding cameras and photos, a listing of various subcategories of topics related to cameras and photos would be presented. From there, further navigation can be made to confine the search until the final result is acquired.

\section{Multilateral (many-to-one)}

The communication relationship is multilateral as it involves many sources to only one target. The information is referred by various sources asynchronously as they access the same target. Although additional target sources may be indicated 
using 'Links', communication is fundamentally between the many-to-one interaction of the sources and the target

\section{Multilateral Logic-driven (C3R1): http://www.starbucks.com}

This organizational target site belongs to Starbucks coffee. The main function is to serve as a promotion platform for the Starbucks Company of their product and to provide consumer information to the public. The information provided is very exclusive, pertaining mostly to the company itself. It is also geared to achieve marketing goals.

Contents: Minimal information is presented to allow a comfortable reading experience. In addition, the information is centered in the middle of the page to catch attention. On the top portion of the page are options that helps find information about domestic store location, international store locations, nutrition values, help, and access to Starbucks account for members. Underneath in bigger fonts are options to access information about the different types of coffee, stores, the homepage and information about the company, etc. Right in the center of the page are advertisements from the Starbuck Company promoting beverages. On the bottom center of the page are hyperlinks to different purchasing options. There are also promotional sale items presented in that portion of the page.

Results: The results are stationary at the target site. Most of the information available cannot be managed in any way. Therefore, not much interaction occurs between the source and the target. It is very much face-valued, obvious and do not require much seeking information. When the option "our coffees" is selected, different types of coffees that the Starbuck Company is promoting are shown along with a brief description of each. There are options to make purchases and also a search bar on the right hand side of the page that looks for different types of coffee not presented on the page. When the option "search" is selected, a page containing only a search bar appears. Information can be inputted to search for specific products. However, what can be searched is limited by Starbucks as the input cannot be more than 30 characters long.

\section{Multilateral Subject-driven (C3R2): http://home.disney.go.com/lite/index}

This organizational target site belongs to Disney Corporations. The main function of this site is for the interaction between the target and its sources, whether it be through events, games, music, movies or shopping. In addition to the promotion of the organization, an emphasis is also put on the epurchase component which allows purchases of Disney products online. The information provided is very exclusive, pertaining mostly to the company itself.

Contents: Along the top portion of the page are options to different genres of information, for example, movies, TV, games, music, events, travel characters, shop, etc. In addition to word links, the target also provides an image link. For example, the "travel" option can be selected either by clicking on the highlight word travel, or by clicking on the castle image. On the upper center of the page is the search bar to directly input inquiries. On the left hand portion of the page are options for different age groups and genders, for example, preschool, boys, girls, teens, etc. In the center of the page are advertisements promoting update news about the target. On the right hand portion of the page are links to top ranking games. Across the bottom portion of the page are different character images.

Results: The results which appear on the target depend largely on the option that was indicated by the source. There are huge amounts of interaction between the source and the target. A few interactive examples include: when a search is made for "communications", a list of relevant topics will be presented and by selecting the "shop" option direction to a more shopping-specified page will be provided.

\section{Multiplex (many-to-many)}

The communication relationship is multiplex as it involves many sources to many targets. The information is communicated amongst various sources with a target that infers further information from other targets. The ultimate goal is to gather trustworthy information that matches the inquiry generated by the various sources. Communication is between the many-to-many interaction of the sources asynchronously to targets with inference engines

\section{Multiplex Logic-driven (C4R1): http://www.howardforums.com}

This target is in the form of a forum which gives its members the opportunity to share knowledge and opinions on various cell phone related topics. Registered members can comment on each other's posts and provide input on various topics. The target is categorized by brands and is then further 
categorized by phone models. Members can also sell cell phone related merchandise to each other.

Contents: The target logo is located on the top left hand corner of the page. On the right hand corner are options that provide information about the target, for example, FAQ's, rules, contacts, and etc. On the upper portion of the page is a tool which members can log into their accounts, or for interested nonmembers to register. In the center portion of the page are different forum topics. Information is provided that tracks the last post in discussion groups thread numbers and post numbers. On the left hand portion of the page are advertisements.

Results: The results are stationary at the target site. However, there are many navigation tools available that helps to browse the target. For example, when the "search" option is selected, direction to a search bar will be provided where specific inquiries may be inputted. When a posting within the discussion section is selected information pertinent to the section like name, price and images will be provided. Options are available on the upper right hand corner that directs to different information regarding the target Underneath the login tool bar are options which provides direction to the register page, the FAQ page, and the search pages, etc. The number listings on the center right hand portion of the site allow skipping around the target. And the highlighted topics for each cell phone discussion sections act as links that directs to different discussion board.

\section{Multiplex Subject-driven (C4R2): http://www.google.com/}

The target "Google.com" serves as a search engine connecting sources to different information available on the web. Sources simply "tell" the engine what they want to look for by inputting the desired "question" and wait for the engine to find all "answers" that are most relevant. The source would then click on the link of they find most helpful to obtain the appropriate information.

Contents: The layout of the target homepage is very basic. In the center top portion of the page is the name of the target search engine (Goggle). In the center portion of the page is the search bar. Different options are available above the search bar to choose the context in which search results can be presented in, for example, "images", "videos", etc. More context options are available on the right hand side of the search bar. On the center bottom portion and the top right hand corner of the page are options that provide information about the target search engine
Results: The results are much tailored to the inquiry of the source, that is, they are displayed only after inquiries have been provided. There are large amount of interactions between the sources and the targets. Results can vary greatly, allowing a lot of different venues within which navigation can take place. A few examples of interaction includes: when "communication" was searched, a list of 'answers' of different separate targets that deal with communication over the Web shows up. The 'answer targets' are presented in the order of being most to least relevant. When "communication" was searched in the image context, a similar listing of relevant targets in the form of images was provided. There are not many navigation tools presented. However, it must be noted that not a lot of navigation tools should be required as the main function of the target is to direct sources to other, more relevant target sites.

\section{CONCLUSIONS}

There are two separate approaches toward the use of the Web; synchronous and asynchronous. Synchronous use of the Web is the extension of traditional communication overcoming a space constraint. Asynchronous use of the Web, however, is the revolutionary communication overcoming both time and space constraints that govern human society for a long time. It enables a business to be up and running 24 hours by 7 days per week throughout the year. As long as a proper network connection is provided, a user can communicate with any web site of his/her interest. This flexible and temporal relationship is produced, reproduced, and transformed over time whenever a user interacts with each web site. Therefore, web communication structure only exists when a user actually experiences the specific web (Orlikowski, 2000, 2002).

In this study, we define the asynchronous web communication further as human-to-web communication, where all types of communicators can be involved in terms of a 'web, such as humans, web servers, database, information systems, etc. Therefore, the source of communication is always a human (user) and the target of communication is information (processed contents and results) in this study, such as to servers, humans, database, information systems, etc.). The benefits of web communication is the effective communication between other users (MultiLateral and MultiPlex), regardless of their time and space constraints. The other important contribution is the conceptualization of new web trends from both syndication of web contents and semantics of web contents. This 
inference model (UniPlex and MultiPlex) is gearing toward more intelligent web communication, by transforming the traditional contents management of reference model (UniLateral and MultiLateral) as the infrastructure of web communication (Eberhart, 2003; Flake et al., 2003).

The web communication structure proposed in this study exists practically and virtually when a user interacts with a web site. Eight structures are identified based on two dimensions: 1) relationship between communicators (source and target) and 2) control scheme of web communication. Our structural analysis can be used to examine how businesses on the Web attempt to control time and space in the interactions with users for the purpose of communicating their intentions in open source culture (O’Mahony and Ferraro, 2007). It can also serve as a foundation for future research into the classification of digital society through digital contents and the effectiveness of those communication efforts by businesses on the Web in the digital world.

\section{REFERENCES}

Berners-Lee, T., Hendler, J., and Lassila, O., "The Semantic Web", Scientific America.com, 2001, May 17.

Campbell-Kelly, M., "Historical reflections: Will the future of software be open source?", Communications of the ACM, 2008, 51, 10, pp. 2123.

Eberhart, A., "Towards semantically enriched business logic", Electronic Commerce Research and Applications, 2003, 2, pp. 288-301

Flake, G. W., Pennock, D. M., and Fain, D. C., "The self-organized web: the Yin to the semantic web's Yang”, IEEE Intelligent Systems, 2003, July/Aug, pp. 72-86

Giddens, A., The constitution of society, Berkely, CA: University of California Press, Berkely, CA, 1984.

Glaser, B. G., \& Strauss, A. L., The discovery of grounded theory: strategies for qualitative research, Aldine Publishing Company, New York, NY, 1967.

Glaser, B. G., Theoretic Sensitivity, The Sociology Press, Mill Valley, 1978.

Leavitt, H. J., "Some effects of certain communication patterns on group performance",
Journal of Abnormal \& Social Psychology, 1951, 46, pp. $38-50$.

O'Mahony, S., and Ferraro, F., "The emergence of governance in an open source community", Academy of Management Journal, 2007, 50, 5, pp. 1079-1106.

O'Reilly, T., What is web 2.0: design patterns and business models for the next generation of software, http://www.oreillynet.com/lpt/a/6228, 2005.

Orlikowski, W. J. and Robey, D., "Information technology and the structuring of organizations", Information Systems Research, 1991, 2, 2, pp. 143169.

Orlikowski, W., "Using technology and constituting structures: A practice lens for studying technology in organizations", Organization Science, 2000, 11, 4, pp. 404-428.

Orlikowski, W., "Knowing in practice: Enacting a collective capability in distributed organizing", Organization Science, 2002, 13, 3, pp. 249-273.

Rogers, E.M., and Kincaid, D.L., Communication Networks; Toward a New Paradigm for Research. Free Press, London, 1981.

Sayah, J. Y., and Zhang, L., "On-demand business collaboration enablement with web services", Decision Support Systems, 2004, 40, pp. 107-127.

Scott, J., Social network analysis: A handbook $\left(2^{\text {nd }}\right.$ ed.), SAGE Publications, London, 2000.

Shadbolt, N., Hall, W., and Berners-Lee, T., "The semantic web revisited", IEEE Intelligent Systems Journal, 2006, May/June.

Shannon, C. E., and Weaver, W., The Mathematical Theory of Communication, University of Illinois Press, Urbana, 1949.

Shaw, M. E., "Group structure and the behaviour of individuals in small groups", Journal of Psychology, 1954, 38(1), pp. 139-149.

Strauss, A. and Corbin, J., Basics of Qualitative Research: Techniques and Procedures for Developing Grounded Theory, Sage Publications, Thousand Oaks, 1998.

Turner, B. A., "Some practical aspects of qualitative data analysis: one way of organising the cognitive processes associated with the generation of grounded 
theory”, Quality and Quantity, 1981, 15, pp. 225-247.

Wasserman, S., and Faust, K., Social Network Analysis: Methods and Applications, Cambridge University Press, New York, 1994. 